\title{
Reflets
}

Revue ontaroise d'intervention sociale et communautaire

\section{Un travail de défrichage : le développement des services en matière de violence faite aux femmes dans le Nord de l'Ontario}

\section{Diane Dupont, Denyse Culligan et Gaëtane Pharand}

Volume 6, numéro 1, printemps 2000

Approches d'intervention : définir et renouveler nos pratiques

URI : https://id.erudit.org/iderudit/026298ar

DOI : https://doi.org/10.7202/026298ar

Aller au sommaire du numéro

Éditeur(s)

Reflets : Revue ontaroise d'intervention sociale et communautaire

ISSN

1203-4576 (imprimé)

1712-8498 (numérique)

Découvrir la revue

Citer cet article

Dupont, D., Culligan, D. \& Pharand, G. (2000). Un travail de défrichage : le développement des services en matière de violence faite aux femmes dans le Nord de l'Ontario. Reflets, 6(1), 128-140. https://doi.org/10.7202/026298ar

Tous droits réservés (C) Reflets : Revue ontaroise d'intervention sociale et communautaire, 2000
Ce document est protégé par la loi sur le droit d'auteur. L'utilisation des services d'Érudit (y compris la reproduction) est assujettie à sa politique d'utilisation que vous pouvez consulter en ligne. 


\section{Un travail de défrichage : le développement des services en matière de violence faite aux femmes dans le Nord de l'Ontario}

Diane Dupont en «partenariat» avec Denyse Culligan du CFFNOO et Gaëtane Pharand du Centre Victoria pour femmes

Au cours des dernières années, le partenariat est devenu une pratique à la mode, parfois convoitée, parfois imposée. Dans la conjoncture économique et politique actuelle, où nous assistons à un désengagement de l'État providence, particulièrement dans la sphère sociale, les organismes accueillent avec une certaine réserve, voire de la méfiance, les partenariats que proposent nos gouvernements. Et avec raison! Pourtant, du côté francophone, cette pratique de partenariat s'avère jusqu'à maintenant très positive pour les organismes de femmes du Nord de l'Ontario. Aussi exigeante que puisse être l'expérience, elle s'avère non seulement un moyen de survie pour les services en français, mais elle a suscité une créativité et un dynamisme nouveaux chez les francophones, particulièrement chez les groupes de femmes.

\section{Contexte historique}

En juillet 1997, le gouvernement provincial annonçait son intention d'investir 27 millions de dollars au cours des quatre prochaines années dans les projets en matière de violence contre 
les femmes. Dans le rapport Prévention de la violence faite aux femmes: un schéma de mise en cuvre, il s'engageait à investir une somme d'argent consacrée au développement de services en français. L'Action ontarienne contre la violence faite aux femmes ${ }^{1} \mathrm{a}$ alors reçu le mandat d'élaborer un plan stratégique de développement des services en français à l'échelle provinciale.

La première étape du mandat fut de mettre sur pied un comité encadreur, constitué d'intervenantes des diverses régions de la province qui œuvrent dans le domaine de la violence faite aux femmes. Au point de départ, le comité a adopté treize principes directeurs qui guident le processus de décision et d'action. Ces

"...treize principes

articulent et

condensent les

pratiques et les revendications des groupes de femmes de l'Ontario..." treize principes articulent et condensent les pratiques et les revendications des groupes de femmes de l'Ontario qui luttent contre la violence faite aux femmes. Ces principes sont les suivants (AOcVF 1998).

1. Les femmes d'expression française de la province ont droit à des services en français en matière de violence contre les femmes, peu importe leur lieu de résidence.

2. Les femmes ont droit à l'accessibilité et à la gratuité des services partout en province.

3. Les services en français à l'intention des victimes de violence contre les femmes doivent être autonomes, libres d'agir et d'être en français et gérés par et pour les femmes francophones.

4. Les services en matière de violence contre les femmes doivent être développés et gérés selon une approche d'analyse féministe en matière de la violence contre les femmes.

5. Les services en matière de violence contre les femmes doivent refléter et desservir les femmes dans leur diversité.

6. Les services en français en matière de violence contre les femmes doivent demeurer communautaires et ne pas être absorbés à l'intérieur d'institutions ou de services de santé, afin de maintenir leur latitude d'action et pour démontrer que la violence n'est pas un problème individuel de santé, mais bien un problème social.

7. Les services en matière de violence contre les femmes doivent être assurés de façon continue par le gouvernement. 
8. Le plan stratégique doit être conçu selon une perspective provinciale globale et non en opposant les intérêts des diverses régions.

9. Les services en matière de violence contre les femmes ne suffisent pas, à eux seuls, à éliminer la violence contre les femmes. Le plan stratégique doit reconnaitre la complexité du problème et viser une intervention globale sur le système social et patriarcal sur lequel repose la violence contre les femmes.

10. La prévention est au centre de notre intervention et comprend l'ensemble des moyens à prendre pour prévenir la violence contre les femmes, sur une base sociétale et non individuelle.

11. Le gouvernement et les fournisseurs de services en français en matière de violence contre les femmes doivent être redevables à la communauté francophone, en reconnaitre le principe et s'engager envers cette communauté.

12. Les services en français en matière de violence contre les femmes doivent bénéficier du financement approprié pour répondre aux besoins des femmes francophones dans toute la province.

13. Toute livraison de services doit être pensée en fonction des droits de chaque femme à l'égalité, la sécurité et la liberté, droits garantis par la Charte canadienne des droits et libertés.

La deuxième étape du mandat a consisté à faire une étude dressant le portrait provincial et régional des services en français existants,

«...pour les régions $d u$ Nord de l'Ontario, l'étude a permis d'identifier plusieurs lacunes dans les services en français en matière de violence contre les femmes». d'identifier les besoins dans les diverses communautés et d'établir les priorités (Brunet 1998). Ainsi, pour les régions du Nord de l'Ontario, l'étude a permis d'identifier plusieurs lacunes dans les services en français en matière de violence contre les femmes. Ces lacunes ont trait soit une absence de services en français dans certaines villes et villages, soit une offre de services réduite et peu adaptée à la réalité des femmes francophones.

Au moment de l'étude, on ne comptait en Ontario que trois centres francophones de lutte contre les agressions à caractère sexuel (Ottawa, Toronto et Sudbury) et quelques services en français offerts par le biais d'agences bilingues dans certaines régions de la province. À Thunder Bay, après plusieurs années de 
«...il devenait

impératif de

développer des services

en matière de violence

qui soient adaptés à la réalité des femmes

francophones

ontariennes de tout le

territoire partant de la région de Sudbury jusqu'à la frontière manitobaine». revendications pour obtenir l'appui financier du gouvernement pour desservir la communauté des femmes francophones, il n'y avait toujours pas de services financés de manière permanente. C'est pourquoi un des premiers projets à recevoir l'aval du comité encadreur dans le cadre de la planification stratégique a été celui de favoriser l'ouverture, en janvier 1998, de trois bureaux à Thunder Bay, Geraldton et Marathon du Centre des femmes francophones du Nord-Ouest de l'Ontario (CFFNOO). Ce projet a aussi reçu l'appui financier de l'Office des affaires francophones.

Par ailleurs, l'étude démontrait que dans les régions du Grand Sudbury, où la population francophone s'élève à $30 \%$, de l'Algoma et du Nord-Ouest, les services en français en matière de violence conjugale sont nettement inadéquats et ne tiennent pas compte de la réalité des francophones. Les agences bilingues qui apportent un soutien aux femmes qui vivent une situation de violence conjugale et les maisons d'hébergement désignées bilingues, faute de ressources financières et peu outillées pour comprendre les réalités des communautés francophones, offrent leurs services en anglais, la langue la plus en demande, car elle est la langue de la majorité. Dans ce contexte, les services en français sont, la plupart du temps, réduits, sinon carrément ignorés. Ainsi, les femmes de ces régions sont obligées, si elles veulent avoir accès aux services, de les recevoir en anglais seulement. Devant un tel choix, certaines femmes préféreront se rendre dans les maisons d'hébergement de l'Est ontarien, d'autres iront au Québec, mais plus grave encore, une majorité choisira de demeurer dans la situation de violence plutôt que de se retrouver dans un milieu et une culture qui leur sont complètement étrangers.

C'est pourquoi il devenait impératif de développer des services en matière de violence qui soient adaptés à la réalité des femmes francophones ontariennes de tout le territoire partant de la région de Sudbury jusqu'à la frontière manitobaine. Lors des discussions sur la planification stratégique dans cette zone, le consensus des membres a permis d'établir deux priorités:

1) la mise sur pied d'une ligne de crise régionale sans frais pour les femmes francophones victimes d'agression sexuelle, de violence conjugale ou d'autres formes d'abus et 
2) le développement de services en matière de violence conjugale qui répondent efficacement aux besoins des femmes francophones de ces régions.

L'expérience du comité encadreur de planification stratégique constitua donc le point de départ pour le travail en partenariat. À ce comité, siégeaient des intervenantes de diverses régions qui ont mis en commun leurs ressources pour articuler un modèle efficace d'intervention à l'échelle provinciale, autre signe de partage et de partenariat. À un niveau plus régional, le partenariat établi entre le CFFNOO, le Centre Victoria pour femmes de Sudbury (CVF) et le Centre Thérèse de Dubreuilville est encore au rendezvous et cherche à répondre aux deux objectifs régionaux, comme nous le verrons dans la suite du texte.

\section{Ligne régionale de crise et d'écoute sans frais}

Depuis 1995, le Centre Victoria et le Centre Thérèse opéraient individuellement une ligne de crise 24 heures par jour et sept jours par semaine pour les femmes victimes d'agression sexuelle. Les statistiques démontraient que les femmes francophones recouraient à la ligne d'écoute pour des problématiques autres que l'agression sexuelle, tout particulièrement pour des situations de violence dans les relations amoureuses. On ne pouvait plus désormais limiter l'offre des services et ignorer ces demandes. Les bénévoles et les travailleuses travaillant à la ligne de crise firent une mise à jour en suivant une formation en violence conjugale. Pendant ce temps, la région du Nord-ouest n'avait aucun accès à une ligne de crise francophone.

Ainsi, dans un premier temps, les trois Centres effectuèrent une étude de faisabilité sur la création d'une ligne régionale qui, tout en tenant compte de l'autonomie des Centres, permettrait une collaboration et une efficacité technique visant à alléger les responsabilités liées à l'opération d'une ligne d'écoute offerte 24 heures par jour et sept jours par semaine. Il fallait également développer des mécanismes pour que les femmes, quelle que soit la provenance de leur appel, puissent bénéficier d'un service efficace. 
"Étant donné que les femmes francophones sont parsemées sur un vaste territoire géographique, il importait que les intervenantes à la ligne soient outillées pour les référer aux ressources adéquates et accessibles dans leurs régions.»
À l'automne 1998, les trois centres inauguraient la nouvelle ligne régionale 1-877-FEM-AIDE. À tour de rôle, chaque centre assure le service de la ligne pendant une semaine. Pour répondre aux besoins urgents d'accompagnement ou pour résoudre les problèmes techniques, une travailleuse dans chacune des régions est sur appel. Par exemple, si le CFFNOO reçoit un appel d'une femme de Sudbury qui vient de vivre une agression sexuelle et qui demande de se faire accompagner à l'hôpital, l'intervenante à la ligne d'écoute contacte la travailleuse sur appel de Sudbury pour que celle-ci se rende à l'hôpital. Un tel mécanisme permet d'assurer un meilleur suivi auprès des femmes.

Étant donné que les femmes francophones sont parsemées sur un vaste territoire géographique, il importait que les intervenantes à la ligne soient outillées pour les référer aux ressources adéquates et accessibles dans leurs régions. Pour ce faire, on a préparé un manuel de ressources régionales. Ce manuel comprend une carte géographique et une liste des ressources pertinentes pour chacune des régions, de sorte que l'intervenante à la ligne puisse repérer rapidement et efficacement ce qui s'offre et répond aux besoins de l'appelante dans sa région. Ce manuel comporte également une section indiquant les principaux repères en intervention de crise afin de soutenir les bénévoles dans leur travail à la ligne de crise et d'écoute.

Cette répartition de la ligne entre les trois centres a l'avantage de libérer du temps et de permettre aux intervenantes de développer d'autres services dans leur région. Il est clair que la ligne de crise constitue un élément complémentaire dans une gamme de services indispensables. C'est pourquoi la plupart des centres offrent, entre autres, de l'intervention individuelle auprès des femmes victimes d'agression sexuelle ou de violence conjugale, de l'accompagnement à la cour ou à l'hôpital, des groupes de soutien, de la référence à d'autres agences de la région sans compter les présentations d'ateliers de sensibilisation et de prévention dans les écoles, les collèges, les universités ou dans d'autres groupes sociaux.

Cette expérience de partenariat entre le CFFNOO,le Centre Victoria et le Centre Thérèse comporte donc plusieurs avantages. 
Un de ces avantages a été de répartir les coûts d'opération de la ligne régionale et de développer des stratégies pour éviter le dédoublement de services. Nous avons, par exemple, développé des outils de promotion de la ligne qui peuvent être utilisés dans les trois régions et les économies en frais de production et d'impression ont permis d'élargir l'éventail des outils de promotion. Un tel travail de collaboration permet également de briser l'isolement des intervenantes, particulièrement celles dans les régions éloignées et d'échanger les ressources et les expertises entre les trois régions. Par exemple, des intervenantes du Centre Victoria sont allées à Thunder Bay donner la formation de base s'adressant aux bénévoles à la ligne de crise aux intervenantes de Thunder Bay, Marathon, Geraldton et Dubreuilville. Par ailleurs, l'intervenante du Centre Thérèse de Dubreuilville est venue à plusieurs reprises à Sudbury pour préparer et former les équipes de bénévoles au programme ESPACE et offrir la formation INSTINCT dans quelques écoles secondaires. À un autre moment, les intervenantes et les bénévoles des trois centres ont pu bénéficier d'une formation commune. Mais d'abord et avant tout, la ligne régionale permet de briser l'isolement des femmes francophones dans les régions plus éloignées.

\section{Le développement d'un modèle de services en matière de violence conjugale}

"...les services

existants dans les

régions pour intervenir

auprès des femmes qui

vivent des situations

de violence conjugale

ne sont pas ou ne sont

que partiellement

accessibles aux femmes

francophones».
Actuellement, les services existants dans les régions pour intervenir auprès des femmes qui vivent des situations de violence conjugale ne sont pas ou ne sont que partiellement accessibles aux femmes francophones. Dans la région de Sudbury-Manitoulin, de l'Algoma et du Nord-ouest, une seule maison d'hébergement est désignée bilingue. Malgré cette désignation, il n'y a aucune garantie que les femmes francophones qui y sont hébergées reçoivent des services en français 24 heures par jour et sept jours par semaine. Et ces services se limitent souvent à l'accueil en maison d'hébergement et encore là, cela dépend souvent de l'heure de son arrivée. Si la femme veut bénéficier d'une intervention ou participer à un groupe de soutien pendant son séjour à la maison d'hébergement, elle n'a pas d'autre choix que de recevoir ces 
"...les compressions

budgétaires dans les

programmes

d'intervention

communautaire privent

de services un nombre

considérable de femmes

vivant dans une

relation abusive». services en anglais, à moins qu'elle ne se déplace vers d'autres agences pouvant répondre à ses besoins.

De plus, dans les dernières années, les programmes d'intervention communautaire des maisons d'hébergement de diverses régions ont cessé faute de subventions. Cet élément est d'autant plus troublant que les statistiques montrent qu'une faible proportion des femmes victimes de violence conjugale choisiront d'aller à une maison d'hébergement. Il en résulte donc que les compressions budgétaires dans les programmes d'intervention communautaire privent de services un nombre considérable de femmes vivant dans une relation abusive.

Un autre facteur à considérer dans le développement de nouveaux modèles de services est le modèle d'hébergement actuel. Si ce modèle convient bien aux milieux urbains où la densité de la population fait en sorte qu'on peut avoir accès aux autres services de prise en charge tels les cliniques juridiques, les bureaux d'assistance sociale, le système de transport organisé, les logements à prix modique, etc., tel n'est pas le cas lorsqu'on est à l'extérieur des centres urbains. De plus, en milieu urbain, l'anonymat des femmes peut être mieux préservé qu'en milieu semi-rural ou rural : celles qui ont un emploi peuvent continuer à se rendre à leur travail, les enfants peuvent continuer à aller à la même école, etc. Ainsi, les restructurations du gouvernement qui ont tendance à centraliser et à urbaniser les services ont pénalisé les populations des régions rurales. Cela est d'autant plus vrai dans le Nord de l'Ontario, puisqu'une forte proportion de la population francophone vit en milieu rural et est dispersée sur un vaste territoire géographique. L'expérience démontre que les gens vivant en milieu rural utilisent peu les services concentrés dans les milieux urbains et cela pour diverses raisons : distances à parcourir, services peu adaptés à leur réalité, attitudes condescendantes à l'égard des personnes vivant en milieu rural, etc.

Dans un tel contexte, l'intervention communautaire (outreach) s'avère indispensable, car plus les femmes sont éloignées des maisons d'hébergement, moins elles y auront recours. De plus, les femmes francophones savent qu'elles ont de fortes chances de se retrouver dans un milieu anglophone peu sensible à leur réalité. 
«Nous privilégions donc un modèle de services décentralisé et flexible dans lequel chacune des régions détermine les mécanismes à mettre en place pour assurer que les femmes francophones victimes de violence reçoivent un soutien adéquat».

«En plus de ces services directs, qui sont offerts sur une base quotidienne, elles préparent et offrent des ateliers de sensibilisation et de prévention auprès d'une clientèle diversifiée...»
Si les femmes hésitent à accéder à des services davantage concentrés dans les milieux urbains, nous avons à développer des mécanismes qui pourront les rejoindre dans leur milieu.

Nous privilégions donc un modèle decentralisé et flexible de services, dans lequel chacune des régions détermine les mécanismes à mettre en place pour assurer que les femmes francophones victimes de violence reçoivent un soutien adéquat. Le partenariat créé entre les trois centres et le travail de concertation ont permis d'apporter aux intervenantes des régions, les ressources et l'appui nécessaires pour travailler auprès des femmes dans leur milieu, de créer un réseau pour briser l'isolement vécu par les femmes et enfin, d'offrir une gamme variée de choix aux femmes qui vivent dans des situations d'abus.

Depuis 1998, le Centre Victoria et le Centre Thérèse collaborent à développer les services en matière de violence faites aux femmes dans quelques communautés. Trois intervenantes communautaires sont présentes dans les communautés de RaysideBalfour et Vallée-Est (région de Sudbury), à Sault Ste-Marie et à Dubreuilville (région d'Algoma). Selon les besoins et les priorités dans les régions, leurs tâches principales consistent à faire de l'intervention directe à court terme auprès des femmes francophones victimes de violence, à accompagner les femmes à la cour, au poste de police, chez le médecin ou à l'hôpital, à animer des groupes de soutien et à offrir de l'assistance pratique aux femmes qui veulent quitter leur situation de violence et reprendre leur vie en main. En plus de ces services directs, qui sont offerts sur une base quotidienne, elles préparent et offrent des ateliers de sensibilisation et de prévention auprès d'une clientèle diversifiée tels les groupes de femmes ainées, les jeunes dans les écoles, les étudiants et étudiantes des collèges et universités, etc. Sur une base continuelle, les intervenantes du projet appuient les centres dans le recrutement, la formation et le soutien des bénévoles à la ligne de crise. Jusqu'à maintenant, les statistiques démontrent que les femmes francophones ont de plus en plus recours à ces nouveaux services qui n'ont été mis sur pied que très récemment.

Le développement de services de liaison communautaire dans les régions de l'Algoma, du Grand Sudbury et du Nord-ouest 
"Notre défi est d'amener le gouvernement à maintenir les services en place et à combler les lacunes dans les autres régions..." nous a aussi amenées à interagir avec plusieurs agences communautaires, à collaborer avec celles qui travaillent directement ou indirectement avec les femmes francophones qui vivent dans une relation abusive, pour combler les lacunes dans le système. Nous sommes présentement à négocier des protocoles d'entente avec les maisons d'hébergement de Sault Ste-Marie et de Wawa pour que les femmes francophones qui demandent à être hébergées puissent recevoir des services en français par l'entremise de l'intervenante présente dans cette région.

Il s'agit donc d'un modèle de services qui s'inspire du principe de la toile d'araignée. Au lieu de concentrer les services dans un seul endroit où les femmes ont à se déplacer pour recevoir l'aide nécessaire, les intervenantes sont présentes dans la communauté, vont vers les femmes et s'adaptent à leur réalité afin de mieux répondre à leurs besoins. Dans ce modèle, seule la gestion administrative est centralisée afin de libérer les intervenantes pour qu'elles consacrent leurs énergies au développement et à l'intervention communautaire. Il revient donc à la coordination d'assurer la gestion, de formuler des politiques issues des expériences sur le terrain, d'assurer la sécurité et de briser l'isolement des intervenantes communautaires en créant des liens entre elles et en favorisant leur réseautage.

Ce modèle n'est présentement qu'à ses débuts. Plusieurs régions du Nord demeurent encore sous-desservies faute de ressources financières. Notre défi est d'amener le gouvernement à maintenir les services en place et à combler les lacunes dans les autres régions et, en particulier dans la région du Nord-ouest, pour que nous puissions poursuivre le développement de services en partenariat, comme nous le faisons présentement pour la ligne régionale.

\section{Le partenariat à l'échelle provinciale}

Cette expérience de partenariat régional s'inscrit dans un partenariat plus large, soit au niveau provincial, avec l'AOcVF. La planification stratégique a permis de consolider un travail de concertation qui avait été amorcé dans les années précédentes. Lors de la mise en place du plan stratégique, les coordonnatrices 
et les intervenantes de divers centres et provenant de diverses régions de la province ont réussi à dépasser les intérêts particuliers de leurs organismes locaux pour se donner une vision provinciale : celle de négocier la répartition du financement afin que les services en matière de violence faite aux femmes comblent les lacunes dans les diverses communautés francophones de la province. Un tel exercice a entraîné l'articulation d'un discours commun qui est à la base des revendications locales ${ }^{2}$.

Un des résultats les plus fructueux de ce travail de collaboration a été la mise sur pied de rencontres interministérielles avec les bailleurs de fonds provinciaux impliqués dans les projets de violence contre les femmes. Depuis déjà deux ans, les membres du comité du plan stratégique de l'AOcVF s'assoient, au moins deux fois par année, à la même table que les divers porte-parole de la Direction générale de la Condition féminine de l'Ontario, de l'Office des affaires francophones, des ministères du Solliciteur général, des Services sociaux et communautaires et du Procureur général pour discuter de l'état des services en français en Ontario et des modèles à développer pour que ces services soient plus redevables à la communauté francophone.

\section{Conclusion}

Les partenariats ont toujours existé. Si cette approche a été privilégiée au cours des dernières années pour remédier aux coupures budgétaires et éviter le dédoublement des services, d'où le scepticisme exprimé par les organismes communautaires, il semble que les francophones y voient là une stratégie efficace non seulement pour assurer la survie des programmes, mais aussi pour développer de nouveaux modèles. En Ontario français, par exemple, en raison de la dispersion géographique de la population, on ne peut pas se permettre de faire la distinction entre les centres qui luttent contre les agressions à caractère sexuel et les maisons d'hébergement, particulièrement quand on sort des milieux urbains. À cause des lacunes dans les divers services en français en 
"Grâce à ce travail de concertation, les groupes de femmes francophones ont fait des pas de géantes dans la dernière décennie». matière de violence faite aux femmes, nos centres doivent offrir une gamme de services qui dépassent leur mandat initial sans compter le travail de revendication à effectuer aux échelles locale, régionale, provinciale et nationale. Grâce à ce travail de concertation, les groupes de femmes francophones ont fait des pas de géantes dans la dernière décennie.

Demeurons toutefois réalistes. Le travail de partenariat peut être, à certains moments, une expérience exigeante où il faut apprendre à composer avec des stratégies et des intérêts parfois divergents entre les individus ou les agences, ce qui n'est pas toujours évident. Quelquefois on se bute à l'affrontement d'idées et d'intérêts au moment de définir les orientations des projets ou d'entreprendre localement des actions concrètes. Le travail de collaboration implique alors une volonté et des habiletés d'écoute, de dialogue, de négociation, de patience, de souplesse et de tolérance.Toutefois, il nourrit la complicité et enrichit les partages. Il rejoint ainsi une certaine manière de faire qui nous est bien familière à nous les femmes. À la longue, la collectivité y gagne en se voyant offrir des services innovateurs.

\section{Bibliographie}

ACTION ONTARIENNE CONTRE LA VIOLENCE FAITE AUX FEMMES (1998). Plan stratégique de développement des services en français en matière de violence contre les femmes, Ottawa, Action ontarienne contre la violence faite aux femmes.

BRUNET, Lucie (1998). Les services en matière de violence faite aux femmes: le défrichage d'un chemin tortueux. Profil des services en français en matière de violence faite aux femmes et identification des défis et des besoins dans la prestation de services en français, Ottawa, Action ontarienne contre la violence faite aux femmes.

\section{Notes}

1. L'AOcVF est un organisme provincial dont les buts sont : la promotion du développement de ressources pour les femmes francophones de l'Ontario, le soutien de l'action des intervenantes par la formation et la réalisation de matériel en français, le développement de la concertation et de la communication entre les groupes communautaires, les agences et les organismes et la prévention de la violence faite aux femmes par la sensibilisation et l'action. 
2. À partir de ce discours, il a été possible de développer des projets divers afin d'adresser immédiatement les besoins les plus pressants. Ces projets sont : la mise sur pied du Centre des femmes francophones du Nord-Ouest de l'Ontario et ses points de services à Thunder Bay, Geraldton et Marathon; le partenariat de la ligne régionale pour les régions de Sudbury à la frontière manitobaine; le développement communautaire dans le domaine de la violence pour l'Algoma et Sudbury; le développement de services directs pour les femmes des minorités raciales et ethnoculturelles dans les villes d'Ottawa, Toronto et Hamilton; la ligne régionale pour le centre, sud et sud-ouest de l'Ontario; les rencontres bi-annuelles des intervenantes francophones dans les centres œuvrant dans le domaine de la violence faite aux femmes, etc. 